\title{
Effects of pre-eclampsia and fetal growth restriction on C-type natriuretic peptide
}

\author{
EA Espiner, ${ }^{\text {a }}$ TCR Prickett, ${ }^{a}$ RS Taylor, ${ }^{b}$ RA Reid, ${ }^{c}$ LM McCowan ${ }^{b}$ \\ ${ }^{a}$ Department of Medicine, University of Otago, Christchurch, New Zealand ${ }^{b}$ Department of Obstetrics and Gynaecology, Faculty of Medical \\ and Health Sciences, University of Auckland, Auckland, New Zealand ${ }^{c}$ Department of Obstetrics and Gynaecology, University of Otago, \\ Christchurch, New Zealand \\ Correspondence: Prof EA Espiner, Department of Medicine, University of Otago, Christchurch, PO Box 4345, Christchurch 8140, \\ New Zealand. Email eric.espiner@otago.ac.nz
}

Accepted 8 February 2015. Published Online 5 April 2015.

\begin{abstract}
Objective To determine changes in plasma C-type natriuretic peptide (CNP), a paracrine product of the vascular endothelium, in pregnancies with vascular disorders, and relate these to time of presentation and severity.
\end{abstract}

Design Retrospective nested cases and controls.

Setting Community study, Auckland New Zealand.

Population Screening for Pregnancy Endpoints (SCOPE) data and bio-bank of maternal plasma.

Methods Maternal plasma amino terminal proCNP (NTproCNP) was measured by radioimmunoassay in early (14-16 weeks of gestation, and again at 19-21 weeks of gestation) and late (34-36 weeks of gestation) pregnancy in three groups of women (20 per group): pre-eclampsia (pre-eclampsia); gestational hypertension (GHT) with small for gestational age (SGA); and uncomplicated pregnancy.

Main outcome measures Change in NTproCNP and associations with concurrent blood pressure, time of case presentation, severity, and infant birthweight.
Results Plasma NTproCNP in early pregnancy in women with vascular disorders did not differ from those found in controls. In late pregnancy, levels in pre-eclampsia $(28.8 \pm 2.3 \mathrm{pM})$ and in GHT with SGA $(28.6 \pm 4.8 \mathrm{pM})$ were significantly increased ( $P=0.01$ and 0.027 , respectively) compared with controls $(21.3 \pm 1 \mathrm{pM})$. In pre-eclampsia, levels were significantly higher $(P<0.03)$ at $14-16$ weeks of gestation in women diagnosed prior to 34 weeks of gestation. Combining all three groups, associations of NTproCNP with concurrent diastolic and mean arterial pressure were found at 34-36 weeks of gestation $(r=0.46)$. No significant associations were identified with birthweight.

Conclusions CNP secretion during gestation is responsive to vascular stress. Plasma NTproCNP measurements may have clinical application in late pregnancy in defining the different phenotypes associated with pre-eclampsia.

Keywords Fetal growth restriction, hypertension, NTproCNP, pre-eclampsia, pregnancy, small for gestational age.

Tweetable abstract Plasma NTproCNP is raised in women diagnosed with PET in a case-controlled study using SCOPE data and biobank.

Please cite this paper as: Espiner EA, Prickett TCR, Taylor RS, Reid RA, McCowan LM. Effects of pre-eclampsia and fetal growth restriction on C-type natriuretic peptide. BJOG 2015; DOI: 10.1111/1471-0528.13397.

\section{Introduction}

C-type natriuretic peptide (CNP) is a paracrine growth factor widely expressed in tissues, including the vascular endothelium, ${ }^{1,2}$ where it is considered to provide vasoprotective functions, ${ }^{3}$ including anti-inflammatory, anti-thrombotic, and anti-proliferative actions in intimal tissues. ${ }^{4}$ In addition, CNP, a vasodepressor, promotes bloodflow via vasodilation and angiogenesis. ${ }^{5,6} \mathrm{CNP}$ itself is rapidly degraded in tissues, and negligible quantities enter the circulation in most species; ${ }^{1}$ however, an inactive portion of the pro-hormone aminoterminal proCNP (NTproCNP) is not degraded at source, circulates in plasma, and can be used as a marker of CNP synthesis in tissues, ${ }^{7}$ provided renal function is normal. ${ }^{8}$ Little is known of the role of CNP in humans but recent studies in pregnant ruminants indicate that maternal concentrations of NTproCNP are regulated during pregnancy, and are increased when maternal nutrients are restricted in early or late gestation. ${ }^{9,10}$ We therefore studied human pregnancy, ${ }^{11}$ postulating that similar increases in maternal plasma NTproCNP would occur when pregnancy was complicated by diminished fetal growth and/or in conditions associated with impaired placental blood flow. The results supported the hypothesis in showing much higher concentrations in complicated pregnancy compared with the lower and relatively unchanging 
NTproCNP levels when pregnancy was uneventful. Disorders of fetal growth and high blood pressure appeared to make the greatest contribution to these elevations, but numbers of women with specific adverse events, such as pre-eclampsia, or gestational hypertension (GHT) plus small for gestational age (SGA), were small in the original publication, prohibiting any analysis of the time sequence of NTproCNP changes or the underlying mechanisms contributing to changes in maternal levels.

We now report serial changes in maternal plasma CNP peptides in two specific complications of late pregnancy pre-eclampsia and GHT with SGA - using samples obtained from the Screening for Pregnancy Endpoints (SCOPE) study. $^{12,13}$ Specifically, we postulated that: (1) compared with controls, NTproCNP concentration in late gestation will be increased in both pre-eclampsia and GHT with SGA; and (2) that observed increases will be associated with appropriate markers of severity. We further postulated that compared with values in women first diagnosed with pre-eclampsia near term, women with early presentation of pre-eclampsia ( $<34$ weeks of gestation) would exhibit higher concentrations of NTproCNP when first assessed in early gestation.

\section{Methods}

\section{Study groups}

Twenty nested cases (pre-eclampsia, GHT with SGA) and controls (uneventful pregnancies) were selected from the SCOPE study (trial registration ACTRN 12607000551493) undertaken in Auckland, New Zealand, between November 2004 and July 2007. Subjects in each of these three groups were selected at random on the basis of previously defined diagnostic end points, ${ }^{12}$ and the availability of plasma samples at the three specified time periods (14-16 weeks of gestation, 19-21 weeks of gestation, and late gestation), as described in Appendix S1. In late gestation, the median time of sampling was 35 weeks of gestation (interquartile range 34-36 weeks of gestation). Of the 20 GHT with SGA, only five had plasma samples collected in late gestation. Briefly stated, SCOPE was a prospective multicentre cohort study of healthy nulliparous women, the primary objective of which was to develop screening tests to predict pre-eclampsia, SGA, and spontaneous preterm birth. All participants were interviewed, and clinical details (including body mass index, BMI, and blood pressure) were recorded at 14-16 and 19-21 weeks of gestation, when blood samples were also collected. Uterine artery Doppler screening was performed in all 60 women at 19-21 weeks of gestation, ${ }^{13}$ and the mean of the right and left uterine artery resistance index was calculated. Women were followed throughout pregnancy, and details of any pregnancy complications and final outcomes were recorded in the SCOPE internet data- base with complete audit trail (MedSciNet ${ }^{\mathrm{AB}}$ ). Whenever possible, clinical details and blood sampling were repeated when the disorder was first recognised (time of diagnosis, see below) for women with pre-eclampsia and GHT with SGA, along with urinary protein/creatinine ratio (cases) or protein dip stick (controls). All blood pressures (diastolic at Korotkoff V) were measured manually in the sitting position by the research midwife using a mercury or aneroid sphygmomanometer, with a large cuff for arm circumferences $\geq 33 \mathrm{~cm}$. Mean arterial pressure (MAP) was derived from the equation $\mathrm{MAP}=\mathrm{DP}+\mathrm{PP} / 3$, where $\mathrm{DP}$ and $\mathrm{PP}$ are diastolic and pulse pressure, respectively. Gestational hypertension (GHT) was defined by blood pressure recordings of systolic pressure $\geq 140 \mathrm{mmHg}$ and/or diastolic pressure $\geq 90 \mathrm{mmHg}$ on at least two occasions, $4 \mathrm{~h}$ apart, after 20 weeks of gestation, but before the onset of labour. Pre-eclampsia was defined as for GHT, plus: (1) proteinuria (24-hour urine protein $\geq 300 \mathrm{mg}$, spot urine protein/creatinine ratio $\geq 30 \mathrm{mg} / \mathrm{mM}$ creatinine, or urine dip-stick protein $\geq 2+$ ); or (2) any multisystem complication of pre-eclampsia. ${ }^{12}$ Severe pre-eclampsia was defined by the presence of sustained severe hypertension (two or more recordings of systolic pressure $\geq 170 \mathrm{mmHg}$ or diastolic pressure $\geq 110 \mathrm{mmHg}$, ${ }^{14}$ or evidence of multisystem disorder. Early-onset pre-eclampsia was defined as pre-eclampsia presenting before 34 weeks of gestation. SGA was defined as a birthweight below the tenth customised centile, adjusted for each of the following: maternal weight, height, parity, ethnicity, infant sex, and gestational age at delivery. The time of diagnosis, defined as the gestational age at which the criteria for pre-eclampsia was first established, also determined the timing of repeat latepregnancy blood collection in all 20 women with preeclampsia. As GHT was not a primary end point in the SCOPE study, diagnosis of GHT with SGA at the time of late pregnancy sampling (34-36 weeks of gestation) could only be confirmed retrospectively once status (SGA, GHT, or uncomplicated pregnancy) was ascertained after birth. In the case of the five women confirmed as GHT with SGA at birth and sampled at 34-36 weeks of gestation, three had GHT plus suspected SGA at the time of sampling, and two were sampled as normal controls who developed GHT after sampling but before delivery. In order to match the timing of sampling in cases, controls were selected who also had additional blood sampling in late gestation (34-36 weeks of gestation).

\section{Specimen collection}

Blood was collected into plain tubes (serum creatinine) or EDTA vacutainer tubes (Bectin Dickinson BD Australia/NZ Auckland NZ), which were then placed on ice and centrifuged at $2400 \mathrm{~g}$ for 10 minutes at $4^{\circ} \mathrm{C}$, then stored at $-80^{\circ} \mathrm{C}$ within 4 hours until assayed for NTproCNP. Plasma NTproCNP was measured as previously described, ${ }^{15}$ 
except that $250 \mu \mathrm{l}$ of plasma was used in the SepPak extraction (concentration) step, and a more sensitive primary antiserum (J39) was used in the assay. Detailed characteristics of the assay are provided in Appendix S2. Within and between assay coefficients of variation were 6.6 and $9.4 \%$, respectively, at $14.5 \mathrm{pmol} / \mathrm{l}$. The detection limit was 3 pmol/l. Cross-reactivity with Atrial Natriuretic Propeptide was $<0.07 \%$, and with human B-type Natriuretic Propeptide was $<0.4 \%$.

Serum creatinine (only assayed in women with preeclampsia at the time of diagnosis) was measured by the Architect c8000 analyser (Abbott Park, IL, USA).

\section{Statistics}

Results are expressed as means \pm SEMs unless stated otherwise in the text. ANOVA with repeated measures was used to assess changes in NTproCNP and blood pressure with time. Bonferroni post hoc analysis was used to detect significant differences between the three time points. Spearman's rank coefficient was used to determine correlations between variables, presented as $r$ values. Statistical significance was assumed when $P<0.05$.

\section{Results}

The demographic and clinical details of the three groups are shown in Table 1. Mean age, BMI, and gestation of late pregnancy blood sampling did not differ significantly among the groups. Gestation was shorter in cases compared with controls. As expected, customised birthweight centile was reduced in GHT with SGA, but not significantly reduced in pre-eclampsia, compared with controls.

\section{Plasma NTproCNP}

Sample collection at $14-16$ weeks of gestation (20 in each group), 19-21 weeks of gestation (20 in each group), and at late gestation (20 in controls and pre-eclampsia, five in GHT with SGA) was complete; however, as two women with pre-eclampsia received high-dose betamethasone within 24 hours before sampling (with the likely suppression of NTproCNP), ${ }^{7}$ results at the time of diagnosis in these two women were excluded from the analysis.

\section{Controls}

As shown in Figure 1(A), the mean maternal plasma levels of NTproCNP at 14-16 weeks of gestation $(17.4 \pm 1.0 \mathrm{pmol} / \mathrm{l})$ and at $19-21$ weeks of gestation $(17.4 \pm 0.8 \mathrm{pmol} / \mathrm{l})$ did not differ. In late pregnancy (34.9 \pm 0.7 weeks of gestation), the mean plasma NTproCNP level $(21.3 \pm 1.0 \mathrm{pmol} / \mathrm{l})$ was significantly increased $(P=0.002)$ compared with values at $14-16$ weeks of gestation, and was positively correlated with gestational age $(r=0.64)$. Neither BMI nor change in BMI (difference between values at 14-16 and 19-21 weeks of gestation) were associated with NTproCNP sampled late in pregnancy. There was no association of late-pregnancy plasma NTproCNP concentration (nor change from values at 1921 weeks of gestation) with customised birthweight centile in women with an uneventful pregnancy.

\section{Women with pre-eclampsia}

Mean plasma NTproCNP at 14-16 weeks of gestation $(18.3 \pm 0.9 \mathrm{pmol} / \mathrm{l})$ and at $19-21$ weeks of gestation $(18.2 \pm 0.8 \mathrm{pmol} / \mathrm{l})$ did not differ from values in controls (Figure 1A). In late pregnancy, plasma NTproCNP

Table 1. Demographic and clinical findings

\begin{tabular}{|c|c|c|c|c|}
\hline Variable & Controls & PE & GHT/SGA & $P$ \\
\hline Ethnicity (\% European) & 80 & 70 & 85 & ns \\
\hline Age (years) & $31.6(0.8)$ & $29.4(0.8)$ & $32.9(0.9)$ & ns \\
\hline Height $(\mathrm{cm})$ & $166(1.7)$ & $165(1.5)$ & $168(1.1)$ & ns \\
\hline \multicolumn{5}{|l|}{ Weight (kg) } \\
\hline 14-16 weeks of gestation & $67.5(1.9)$ & $73.0(3.0)$ & $78.6(5.0)$ & ns \\
\hline 19-21 weeks of gestation & $69.0(2.0)$ & $74.5(2.9)$ & $81.5(5.1)$ & ns \\
\hline \multicolumn{5}{|l|}{ BMI } \\
\hline 14-16 weeks of gestation & $24.4(0.7)$ & $26.7(1.0)$ & $27.7(1.8)$ & ns \\
\hline 19-21 weeks of gestation & $24.9(0.7)$ & $27.3(1.0)$ & $28.7(1.8)$ & ns \\
\hline Gestation at sampling** (weeks) & $34.9(0.7)$ & $35.1(0.7)$ & $34.5(0.9)^{*}$ & ns \\
\hline Gestation at delivery (weeks) & $40.4(0.2)$ & $36.2(0.6)$ & $38.5(0.4)$ & $<0.001$ \\
\hline Customised birthweight centile & $47.5(5.9)$ & $40.6(8.2)$ & $3.6(0.7)$ & $<0.001$ \\
\hline
\end{tabular}



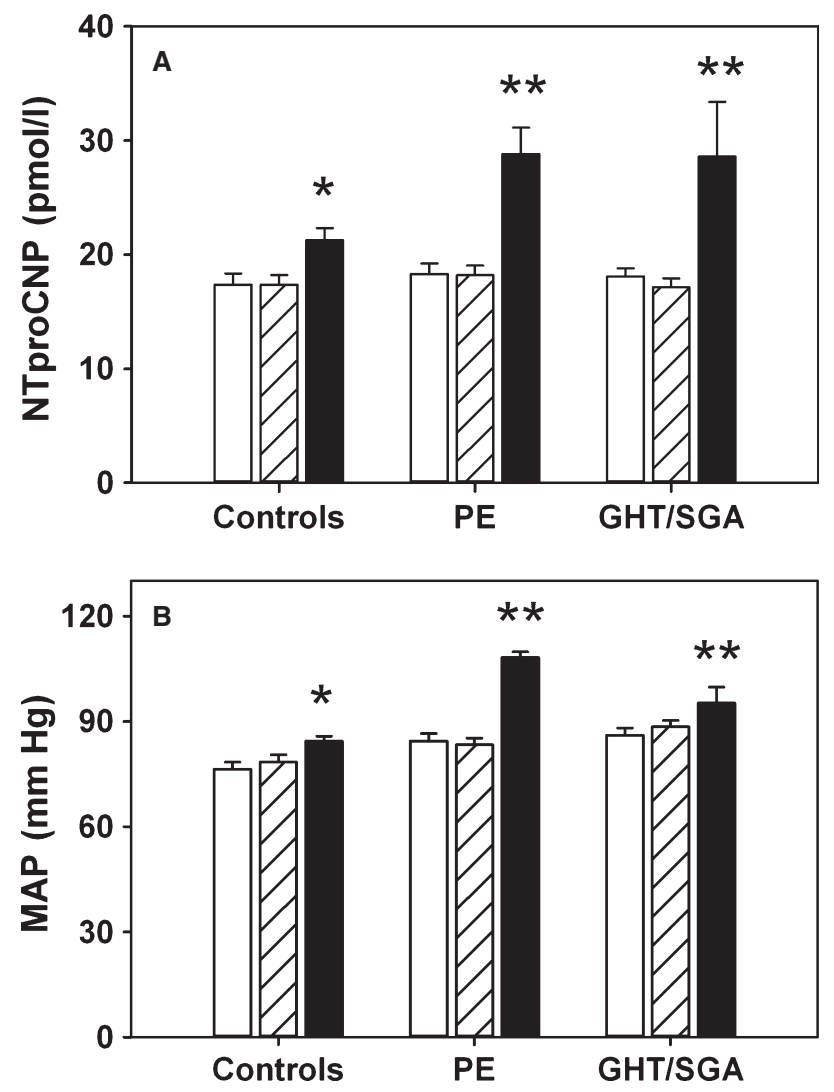

Figure 1. Changes in (A) plasma NTproCNP and (B) mean arterial pressure (MAP) during pregnancy. Open, stippled, and filled columns depict values at 14-16, 19-21, and 34-36 weeks of gestation, respectively. Data are expressed as means \pm SEMs from 20 women at each time point, except for pre-eclampsia $(n=18)$ and for GHT with SGA $(n=5)$ at 34-36 weeks of gestation. Significant differences from the values at 14-16 weeks of gestation are indicated by asterisks: $* P<0.05 ; * * P<0.001$.

$(28.8 \pm 2.3 \mathrm{pmol} / \mathrm{l})$ was significantly higher than control values at the same gestational age $(P=0.01)$, and unlike controls showed no association with gestation. Viewed in relation to severity, mean values in women graded as having severe pre-eclampsia $(28.7 \pm 2.7 \mathrm{pmol} / \mathrm{l}, n=9)$ did not significantly differ from those in women graded as 'not severe pre-eclampsia' ( $28.9 \pm 3.9 \mathrm{pmol} / \mathrm{l}, n=9)$; however, in six women presenting with early pre-eclampsia (at a mean of 31.4 weeks of gestation), plasma NTproCNP at $14-16$ weeks of gestation $(21.3 \pm 1.9 \mathrm{pmol} / \mathrm{l})$ was significantly higher than values in women with later presentations $(17.0 \pm 0.9 \mathrm{pmol} / \mathrm{l}, n=14, P=0.03)$. Similarly, MAP at 14-16 weeks of gestation in these early presentations (mean $93 \pm 3 \mathrm{mmHg}, n=6$ ) was significantly higher than in women presenting after 34 weeks of gestation (mean $81 \pm 2 \mathrm{mmHg}, n=14, P<0.01)$. Neither BMI at $14-$ 16 weeks of gestation nor change at 19-21 weeks of gestation was associated with plasma NTproCNP at any time point. Six of these 20 women with pre-eclampsia delivered an infant that was SGA. Values at time of diagnosis in these women with pre-eclampsia and SGA did not differ from women with pre-eclampsia without SGA. Mean serum creatinine $(67.2 \pm 3.2 \mathrm{mmol} / \mathrm{l}$, range $46-90 \mathrm{mmol} / \mathrm{l})$ was within the normal range at the time of diagnosis sampling in all cases, and showed no correlation with concurrent plasma NTproCNP concentrations.

\section{Women with GHT and SGA}

As shown in Figure 1(A), mean maternal NTproCNP at 14-16 weeks of gestation $(18.1 \pm 0.7 \mathrm{pmol} / \mathrm{l})$ and at $19-$ 21 weeks of gestation $(17.1 \pm 0.8 \mathrm{pmol} / \mathrm{l})$ did not differ, and were similar to time-matched values in controls and women with pre-eclampsia. No significant associations of NTproCNP were found at these times with BMI, nor with customised birthweight centiles. Mean plasma NTproCNP in late pregnancy $(28.6 \pm 4.8 \mathrm{pmol} / \mathrm{l})$ was increased compared with controls $(P=0.027)$ at the same GA. Clinically relevant details are shown for these five women in Table 2. Although high blood pressure was documented during pregnancy in all five women, only two (cases 1 and 5, Table 2) were hypertensive at the time of sampling, late in pregnancy. The women for whom NTproCNP was not raised (17.2 pmol/l, case 1, Table 2) had experienced severe asthma requiring increasingly higher daily doses of inhaled corticosteroids from 14-16 weeks of gestation. Case 5 (exhibiting the highest plasma NTproCNP concentration of $44.4 \mathrm{pmol} / \mathrm{l}$ at the time of diagnosis) developed proteinuria (1+) and hypertension (134 mmHg systolic and $92 \mathrm{mmHg}$ diastolic, the highest values recorded in her pregnancy) at 35 weeks of gestation. She proceeded to deliver an SGA infant at 37.3 weeks of gestation.

\section{NTproCNP and blood pressure}

Blood pressure recordings in the three groups are detailed in Table S1, and associations with concurrent maternal plasma NTproCNP levels are provided in Table S2. The time-related changes in MAP in relation to NTproCNP in each group are shown in Figure 1(B). At the time of sampling in late pregnancy, eight of the 20 women with preeclampsia, but none of the others, were receiving hypotensive therapy.

Blood pressure increased as pregnancy advanced in all three groups (Figure 1B; Table S1). In addition, compared with the levels in controls, pressures were significantly increased at 19-21 weeks of gestation in GHT with SGA. Late in pregnancy, blood pressures were significantly higher in pre-eclampsia compared with those in GHT with SGA $(P<0.005$ for all, Table S1), yet plasma NTproCNP concentration at the time of diagnosis in the two groups did not differ (Figure 1).

Significant correlations of concurrent NTproCNP values with diastolic $(r=0.56)$ and mean arterial pressure 
Table 2. Blood pressure, birthweight, and plasma NTproCNP in five women with GHT/SGA

\begin{tabular}{|c|c|c|c|c|c|}
\hline Case & $\begin{array}{c}\text { Max } \\
\text { SBP* } \\
(\mathrm{mmHg})\end{array}$ & $\begin{array}{c}\text { Max } \\
\text { DBP* } \\
(\mathrm{mmHg})\end{array}$ & $\begin{array}{c}\text { Time of } \\
\text { onset } \\
\text { (weeks of } \\
\text { gestation) }\end{array}$ & $\begin{array}{c}\text { CBWC** } \\
\%\end{array}$ & $\begin{array}{c}\text { NTproCNP*** } \\
(p m o l / l)\end{array}$ \\
\hline 1 & 150 & 100 & 33.0 & 6.0 & 17.2 \\
\hline 2 & 140 & 92 & 33.4 & 1.0 & 34.2 \\
\hline 3 & 150 & 95 & 34.1 & 5.0 & 23.4 \\
\hline 4 & 140 & 100 & 37.0 & 0.4 & 23.7 \\
\hline 5 & 132 & 92 & 35.0 & 5.0 & 44.4 \\
\hline
\end{tabular}

$(r=0.54)$ were observed in controls late in pregnancy (Table S2), but not at other times. In pre-eclampsia, associations were significant $(r=0.49)$ at $14-16$ weeks of gestation (diastolic), and at the time of diagnosis (MAP, $r=0.46$ ), whereas in GHT with SGA no significant association was evident at any time point. Excluding women with pre-eclampsia who were receiving hypotensive drugs (methyl dopa alone in seven women; methyl dopa plus labetalol in one woman) further strengthened the association of plasma NTproCNP with diastolic pressure $(r=0.66)$ and with MAP $(r=0.69)$.

For all groups combined $(n=43$, i.e. 18 women with pre-eclampsia, plus 20 controls, plus five women with GHT and SGA) there was an association of time-matched plasma NTproCNP late in pregnancy with diastolic pressure (Figure 2) and with MAP ( $r=0.46$, for both). The association with systolic pressure was much weaker $(r=0.28)$. Excluding women with pre-eclampsia who were receiving hypotensive therapy did not affect these relationships. Despite associations of MAP with NTproCNP, no significant association of change in plasma NTproCNP across gestation with change in MAP was evident in any of the three groups or in the combined cohort.

Uterine artery resistance index (UARI) and NTproCNP At 19-21 weeks of gestation, mean UARI in controls $(0.55 \pm 0.02)$, pre-eclampsia $(0.58 \pm 0.03)$, and GHT with SGA $(0.58 \pm 0.03)$ did not differ significantly. Associations between UARI and concurrent plasma NTproCNP values did not attain significance in any of the three groups.

\section{Discussion}

\section{Main findings}

Using plasma sampled longitudinally from selected cases in the SCOPE study of nulliparous women, we show that

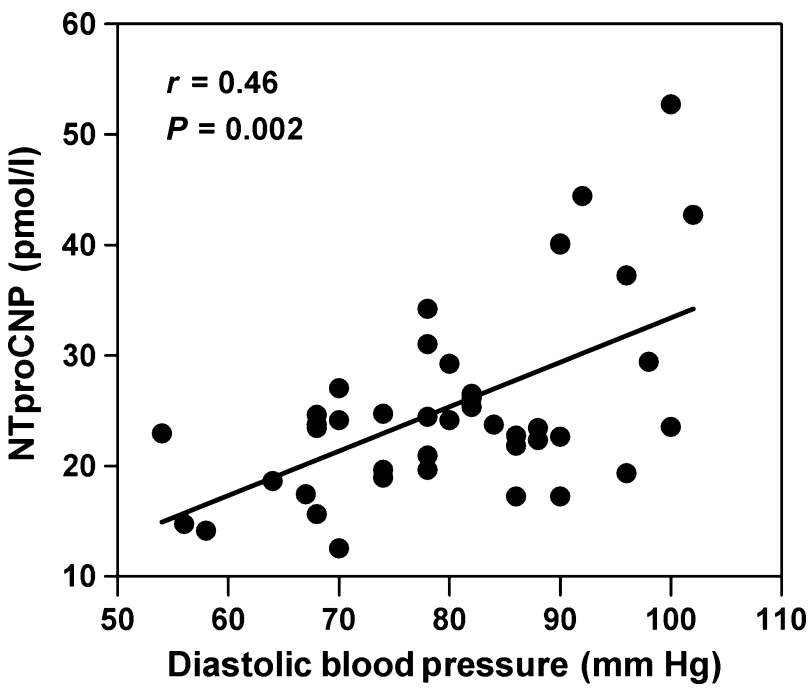

Figure 2. Association of diastolic blood pressure with maternal plasma NTproCNP at 34-36 weeks of gestation $(n=43)$. Line fitted by least squares method.

compared against controls with uneventful pregnancies, maternal plasma NTproCNP is significantly raised in women with pre-eclampsia at the time of diagnosis, and is similarly increased in a smaller number of women with GHT who delivered SGA infants. In addition, we identify novel associations of maternal NTproCNP with concurrent blood pressure at the time of sampling in late pregnancy, and significantly higher values in early gestation in women with pre-eclampsia presenting prior to 34 weeks of gestation.

As found previously, ${ }^{11}$ plasma NTproCNP concentrations show a modest increase in late gestation in uneventful pregnancy. No association was found with maternal blood pressure, height, or BMI early in gestation in normal pregnancies; however, in late gestation a significant positive association of NTproCNP was found with diastolic blood pressure and MAP, even though blood pressure recordings were consistently within normal limits throughout gestation in this group. In contrast, there was no correlation with customised birthweight centile at any time point in women with infants of birthweight appropriate for gestational age. Compared with controls, the mean values of maternal plasma NTproCNP in GHT with SGA did not differ in early gestation, even though blood pressures were significantly higher than in controls at 19-21 weeks of gestation. Viewed in relation to the positive association of blood pressure with NTproCNP in controls late in gestation, these findings suggest that the impact of rising blood pressure on maternal NTproCNP is greater in late gestation. Increases in NTproCNP in late pregnancy in both groups of cases coincided with a further rise in blood pressure that was significantly higher in women with pre-eclampsia. 
In contrast to the above associations of NTproCNP with blood pressure in pre-eclampsia, the clinical grading of the severity of pre-eclampsia or the delivery of an SGA infant was unrelated to plasma NTproCNP. In women with preeclampsia presenting before 34 weeks of gestation, however, NTproCNP was significantly increased at 14-16 weeks of gestation when compared with values from women with later presentations. Another relevant issue to consider is renal function, which declines in pre-eclampsia. Plasma NTproCNP is strongly affected by renal function, even when plasma creatinine values lie within the accepted normal range; $^{8}$ however, the lack of any correlation of NTproCNP with serum creatinine in women who develop pre-eclampsia in late gestation indicates that the observed changes in NTproCNP are unlikely to be based on declining GFR alone, but rather reflect the increasing demands made on the maternal vasculature.

In women with GHT plus SGA (but without evidence of pre-eclampsia or other adverse events), NTproCNP levels do not differ from control values at either 14-16 or 19-21 weeks of gestation, but increase in late pregnancy (34-36 weeks of gestation) to values similar to pre-eclampsia, and exceed those in controls at the same gestational age. As only five women with GHT plus SGA had blood sampled in late pregnancy, these findings are preliminary, but are virtually identical to the results we have previously reported. ${ }^{11}$ In the current study NTproCNP was unrelated to blood pressure at any time point in GHT with SGA. Taken together with the finding that MAP in GHT with SGA was significantly lower than in women with preeclampsia at the time of diagnosis, yet maternal NTproCNP in the two groups did not differ, the results suggest that additional factors arising from restricted fetal growth may also contribute to elevated maternal levels in late gestation, and now need further study.

\section{Strengths and limitations}

Strengths include longitudinal sampling at standardised times across pregnancy, strictly pre-defined end points, and the use of recently developed novel and fully validated approaches to measuring CNP products in maternal plasma. Together, these factors have uncovered associations of CNP products with mean arterial and diastolic pressure in late pregnancy, and higher values early in pregnancy in the less common but more serious pre-eclampsia presenting prior to 34 weeks of gestation. Limitations relate to the numbers of women studied, particularly those sampled with GHT at 34-36 weeks of gestation, and who later delivered an infant with SGA. Although the findings lack immediate clinical application, evidence that vascular complications in pregnancy are associated with the increased secretion of an endogenous vasoprotective peptide with anti-inflammatory actions is an important advance that could lead to improved definitions of pre-eclampsia phenotypes, ${ }^{16}$ or to therapeutic applications now that CNP agonists are approved for use in humans (ClinicalTrials.gov NCT02055157).

\section{Interpretation}

Although CNP has been viewed as an important (paracrine) regulator of vascular tone and inflammation, ${ }^{3-6}$ little is known of circulating CNP products in pregnant women with vascular complications. Moreover, there are few if any reports of raised CNP products in other hypertensive settings, either in humans or in experimental animals. Cheung and Brown studied 19 normal women and age- and gender-matched women with essential hypertension. ${ }^{17}$ Whereas a significant increase in plasma B-type natriuretic peptide (BNP) was observed, plasma CNP did not differ between the two groups. In human pregnancy, Stepan et al. found that maternal plasma CNP concentration in women with pre-eclampsia or GHT did not differ from normal pregnancy values when measured at 26-39 weeks of gestation. ${ }^{18}$ The latter finding is not surprising given the rapid degradation of CNP and the poor correlation with concurrent plasma levels of NTproCNP in human pregnancy. ${ }^{11}$ Despite these negative findings, evidence ex vivo shows that shear stress, likely to increase with the increasing haemodynamic load in pregnancy, ${ }^{19}$ upregulates NPPC gene expression and increases CNP protein production. Connecting these findings is the greatly augmented and selective up-regulation of the CNP receptor (NPRB) and content of CNP in the ovine uterine arterial endothelium in late gestation. ${ }^{20} \mathrm{It}$ is not known whether similar changes in CNP pathway activity occur in the human uterine vasculature during pregnancy, but the current findings linking maternal circulating CNP products with increasing haemodynamic stress in late gestation make this an attractive hypothesis. Of note, an increase in the cardiac natriuretic peptides atrial natriuretic peptide (ANP) and BNP also occur in the third trimester in pre-eclampsia, ${ }^{21,22}$ possibly in response to increased intracardiac pressure. ${ }^{21}$ Correlations of maternal plasma levels of ANP or BNP products with systemic pressure have been inconsistent, however. As production of NTproCNP from the heart is trivial compared with ANP and $\mathrm{BNP},{ }^{23}$ vascular rather than cardiac contributions are the more likely source of the marked increase observed in the current study.

\section{Conclusion}

At the time of first detection, two major complications of late pregnancy - pre-eclampsia and GHT with SGA - are associated with raised maternal plasma NTproCNP. Having identified CNP products as participants in the adaptive response to vascular stress, it is now important to assess its 
clinical utility relative to other analytes in larger groups of women. A number of circulating factors are currently viewed as potentially useful biomarkers of pre-eclampsia and/or SGA, ${ }^{24,25}$ but none has yet been shown to predict late-onset disease when sampled in early pregnancy. ${ }^{16}$ There is an emerging consensus that the phenotype in preeclampsia is quite variable, ${ }^{24}$ making it likely that several different late-pregnancy biomarkers, each reflecting a different disturbed pathology, will be more effective than any single entity in predicting complications in late gestation. NTproCNP, as a marker of maternal vascular stress, could complement promising placental products and improve the detection of women at risk when measured in late pregnancy.

\section{Disclosures}

None.

\section{Contributions to authorship}

$\mathrm{EE}$ and RR designed the study, analysed results and with the assistance of LM wrote the manuscript. RT and LM selected the women and supplied all relevant clinical details from the SCOPE data base. TP supervised all measurements of NTproCNP, performed all statistical analyses and assisted with manuscript preparation and interpretation of results.

\section{Details of ethics approval}

Ethical approval was obtained from the local Ethics Committee (NZ AKX/02/00/364) and all women gave informed consent.

\section{Funding}

This study was supported in part by a grant from Otago Innovation Limited, New Zealand. The SCOPE study was supported by the New Enterprise Research Fund, Foundation for Research Science and Technology; Health Research Council 04/198; and the Evelyn Bond Fund, Auckland District Health Board Charitable Trust.

\section{Acknowledgements}

We would like to thank the pregnant women who participated in the SCOPE Study and the database and statistical support provided by Ms EHY Chan, University of Auckland.

\section{Supporting Information}

Additional Supporting Information may be found in the online version of this article:

Appendix S1. Selection process for cases and controls.

Appendix S2. Christchurch NTproCNP radioimmunoassay.

Table S1. Blood pressure during pregnancy.
Table S2. Associations ( $r$ values) of blood pressure with concurrent plasma NTproCNP.

\section{References}

1 Potter LR, Abbey-Hosch S, Dickey DM. Natriuretic peptides, their receptors, and cyclic guanosine monophosphate-dependent signaling functions. Endocr Rev 2006;27:47-72.

2 Sellitti DF, Koles N, Mendonca MC. Regulation of C-type natriuretic peptide expression. Peptides 2011;32:1964-71.

3 Komatsu Y, Itoh H, Suga Sl, Ogawa Y, Hama N, Kishimoto I, et al. Regulation of endothelial production of C-type natriuretic peptide in coculture with vascular smooth muscle cells: role of the vascular natriuretic peptide system in vascular growth inhibition. Circ Res 1996;78:606-14.

4 Qian JY, Haruno A, Asada Y, Nishida T, Saito Y, Matsuda T, et al. Local expression of C-type natriuretic peptide suppresses inflammation, eliminates shear stress-induced thrombosis, and prevents neointima formation through enhanced nitric oxide production in rabbit injured carotid arteries. Circ Res 2002;91:1063-9.

5 Honing ML, Smits P, Morrison PJ, Burnett JC Jr, Rabelink TJ. C-type natriuretic peptide-induced vasodilation is dependent on hyperpolarization in human forearm resistance vessels. Hypertension 2001;37:1179-83

6 Doi K, Itoh H, Nakagawa O, Igaki T, Yamashita J, Chun TH, et al. Expression of natriuretic peptide system during embryonic stem cell vasculogenesis. Heart Vessels 1997;(Suppl 12):18-22.

7 Prickett TCR, Lynn AM, Barrell GK, Darlow BA, Cameron VA, Espiner EA, et al. Amino-terminal proCNP: a putative marker of cartilage activity in postnatal growth. Pediatr Res 2005;58:334-40.

8 Prickett TC, Olney RC, Cameron VA, Ellis MJ, Richards AM, Espiner EA. Impact of age, phenotype and cardio-renal function on plasma C-type and B-type natriuretic peptide forms in an adult population. Clin Endocrinol (Oxf) 2013;78:783-9.

9 Madhavan S, Prickett T, Ridgway M, Wellby M, Espiner E, Barrell G. Nutrient Restriction in Early Ovine Pregnancy Increases Maternal Plasma C-Type Natriuretic Peptide Concentration: An Adaptive Response to Fetal Growth Restriction. Medical Sciences Congress. Queenstown, New Zealand; 2013. p. 31 Abstract 3B.3.

10 McNeill BA, Barrell GK, Ridgway MJ, Wellby MP, Prickett TC, Espiner EA. Caloric restriction, but not caloric loading, affects circulating fetal and maternal C-type natriuretic peptide concentrations in late ovine gestation. Reprod Fert Develop 2012;24:1063-70.

11 Reid RA, Prickett TC, Pullar BE, Darlow BA, Gullam JE, Espiner EA. C-type natriuretic peptide in complicated pregnancy: increased secretion precedes adverse events. I Clin Endocrinol Metab 2014;99:1470-8.

12 North RA, McCowan LM, Dekker GA, Poston L, Chan EH, Stewart AW, et al. Clinical risk prediction for pre-eclampsia in nulliparous women: development of model in international prospective cohort. BMJ 2011;342:d1875

13 Groom KM, North RA, Stone PR, Chan EH, Taylor RS, Dekker GA, et al. Patterns of change in uterine artery Doppler studies between 20 and 24 weeks of gestation and pregnancy outcomes. Obstet Gynecol 2009;113:332-8.

14 Brown MA, Hague WM, Higgins J, Lowe S, McCowan L, Oats J, et al. The detection, investigation and management of hypertension in pregnancy: executive summary. Aust N Z J Obstet Gynaecol 2000;40:133-8.

15 Prickett TCR, Yandle TG, Nicholls MG, Espiner EA, Richards AM Identification of amino-terminal pro-C-type natriuretic peptide in human plasma. Biochem Biophys Res Commun 2001;286:513-7. 
Espiner et al.

16 Kenny LC, Black MA, Poston L, Taylor R, Myers JE, Baker PN, et al. Early Pregnancy Prediction of Preeclampsia in Nulliparous Women, Combining Clinical Risk and Biomarkers: the Screening for Pregnancy Endpoints (SCOPE) International Cohort Study. Hypertension 2014;64:644-52.

17 Cheung BM, Brown MJ. Plasma brain natriuretic peptide and C-type natriuretic peptide in essential hypertension. I Hypertens 1994;12:449-54.

18 Stepan H, Faber R, Walther D, Walther T. C-type natriuretic peptide levels in women with gestational hypertension and preeclampsia. Obstet Gynecol 1999;93:199-202.

19 Sprague B, Chesler NC, Magness RR. Shear stress regulation of nitric oxide production in uterine and placental artery endothelial cells: experimental studies and hemodynamic models of shear stresses on endothelial cells. Int J Dev Biol 2010;54:331-9.

20 Itoh H, Bird IM, Nakao K, Magness RR. Pregnancy increases soluble and particulate guanylate cyclases and decreases the clearance receptor of natriuretic peptides in ovine uterine, but not systemic, arteries. Endocrinology 1998;139:3329-41.
21 Sugulle $M$, Herse F, Hering $L$, Mockel $M$, Dechend R, Staff $A C$. Cardiovascular biomarker midregional proatrial natriuretic peptide during and after preeclamptic pregnancies. Hypertension 2012;59:395-401.

22 Tihtonen KM, Koobi T, Vuolteenaho O, Huhtala HS, Uotila JT. Natriuretic peptides and hemodynamics in preeclampsia. Am J Obstet Gynecol 2007;196:328.e1-7.

23 Palmer SC, Prickett TC, Espiner EA, Yandle TG, Richards AM. Regional release and clearance of c-type natriuretic peptides in the human circulation and relation to cardiac function. Hypertension 2009;20:612-8.

24 Myatt L, Webster RP. Vascular biology of preeclampsia. J Thromb Haemost 2009;7:375-84.

25 Chappell LC, Duckworth S, Seed PT, Griffin M, Myers J, Mackillop L, et al. Diagnostic accuracy of placental growth factor in women with suspected preeclampsia: a prospective multicenter study. Circulation 2013;5:2121-31. 